\title{
CLOSED-FORM 2D ANGLE ESTIMATION WITH A SPHERICAL ARRAY VIA SPHERICAL PHASE MODE EXCITATION AND ESPRIT
}

\author{
Roald Goossens and Hendrik Rogier \\ Ghent University \\ Department of Information Technology \\ St-Pietersnieuwstraat 41, 9000 Ghent, Belgium
}

\begin{abstract}
We present a new spherical ESPRIT algorithm, being a closed-form algorithm for use in conjunction with spherical arrays that provides automatically paired source azimuth and elevation estimates. It is a numerically efficient $2 \mathrm{D}$ angle-ofarrival estimation algorithm which does not rely on extensive spectral searches or iterative solutions to multi-dimensional optimization problems. The spherical ESPRIT is based on the spherical phase mode excitation principle, enabling us to exploit the spherical symmetry of the antenna array by relying on a recursive relationship between spherical harmonics.
\end{abstract}

Index Terms - Spherical antennas, Direction of arrival estimation, Fourier transforms

\section{INTRODUCTION}

Estimating the directions of arrival (DOAs) of propagating plane waves is a popular subject of research in a variety of applications. Several algorithms are already developed, where the accuracy of the estimates and the computational efficiency are the most important concerns. MUSIC [1] delivers accurate results, but often requires a high computational cost. Only for particular array configurations, such as uniform linear arrays and uniform circular arrays (UCAs), there exist numerically efficient MUSIC-based estimation algorithms. However, these algorithms are often limited to one dimension. ESPRIT [2] overcomes this problem, because it is a closed-form DOA estimation algorithm that provides automatically paired source azimuth and elevation angle estimates. The ESPRIT algorithm relies on a displacement invariance array structure, such that only some specific array configurations are appropriate for applying ESPRIT. In [3] an ESPRIT-based algorithm is developed for UCAs. UCAs are attractive array configurations since the circular symmetry in the array can be exploited by performing a phase mode excitation, consisting essentially of a Fourier analysis. In this paper an ESPRIT-based algorithm is developed for spherical array configurations. Spherical arrays possess a high degree of symmetry and this can be exploited by spherical phase mode excitation [4]. Similar to UCAs, this excitation is now essentially a spherical Fourier transform. By means of a recursive relationship between spherical harmonics an automatically paired azimuth and elevation estimation algorithm is developed.

\section{SPHERICAL ARRAY GEOMETRY AND MANIFOLD}

We consider a spherical antenna array consisting of $N$ identical antenna elements. The position vector of antenna $n$ of the array is $\mathbf{p}_{n}=\left(R \cos \varphi_{n} \sin \theta_{n}, R \sin \varphi_{n} \sin \theta_{n}, R \cos \theta_{n}\right)$. Each antenna element is unambiguously defined by the elevation angle and azimuth angle $\left(\theta_{n}, \varphi_{n}\right)$. The elevation angle is measured down from the $z$ axis and the azimuth angle is measured counterclockwise from the $x$ axis. The choice of $\left(\theta_{n}, \varphi_{n}\right)(n=1, \ldots, N)$ is a complex problem and an appropriate choice will be discussed when the spherical phase mode excitation is considered (see Section 4). Consider a narrowband plane wave with wave number $k_{0}=2 \pi / \lambda$ propagating in the direction $-\mathbf{r}$ with elevation and azimuth angle $\theta$ and $\varphi$, respectively. The unit vector $\mathbf{r}$ has cartesian coordinates $\mathbf{r}=(\cos \varphi \sin \theta, \sin \varphi \sin \theta, \cos \theta)$. The phase difference between the complex envelopes of the signals received at the origin and at element $n$ at a given time is $\psi_{n}=e^{j k_{0} \mathbf{r} \cdot \mathbf{p}_{n}}=e^{j k_{0} R\left(\sin \theta \sin \theta_{n} \cos \left(\varphi-\varphi_{n}\right)+\cos \theta \cos \theta_{n}\right)}$. The element space spherical array manifold vector is therefore

$$
\mathbf{a}(\theta, \varphi)=\left[e^{j k_{0} \mathbf{r} \cdot \mathbf{p}_{1}}, e^{j k_{0} \mathbf{r} \cdot \mathbf{p}_{2}}, \ldots, e^{j k_{0} \mathbf{r} \cdot \mathbf{p}_{n}}\right]^{T} .
$$

\section{SPHERICAL PHASE MODE EXCITATION WITH A CONTINUOUS APERTURE}

Consider a continuous spherical aperture of radius $R$. The normalized far-field pattern produced by the excitation function $w\left(\theta^{\prime}, \varphi^{\prime}\right)$ is $f(\theta, \varphi)=\frac{1}{4 \pi} \iint w\left(\theta^{\prime}, \varphi^{\prime}\right) e^{j k_{0} \mathbf{r} \cdot \mathbf{p}^{\prime}} \mathrm{d} \Omega^{\prime}$, where $\mathbf{p}^{\prime}=\left(R \cos \varphi^{\prime} \sin \theta^{\prime}, R \sin \varphi^{\prime} \sin \theta^{\prime}, R \cos \theta^{\prime}\right)$. Consider a spatial spherical harmonic excitation of the form $w\left(\theta^{\prime}, \varphi^{\prime}\right)=$ $Y_{l, m}\left(\theta^{\prime}, \varphi^{\prime}\right)$, where $Y_{l, m}(\theta, \varphi)$ is defined by

$$
Y_{l, m}(\theta, \varphi)=\sqrt{\frac{(2 l+1)(l-m) !}{4 \pi(l+m) !}} e^{j m \varphi} P_{l}^{m}(\cos \theta),
$$


where $P_{l}^{m}(\cos \theta)$ is the associated Legendre function. The associated Legendre function is defined by:

$$
\left\{\begin{array}{l}
P_{l}^{m}(\cos \theta)=\sin ^{m} \theta \frac{\mathrm{d}^{m} P_{l}(\cos \theta)}{\mathrm{d} \cos m} \theta \quad \text { for } m \geq 0 \\
P_{l}^{m}(\cos \theta)=(-1)^{m} \frac{(l+m) !}{(l-m) !} P_{l}^{-m}(\cos \theta) \text { for } m<0
\end{array}\right.
$$

The corresponding far-field pattern can be expressed as

$$
f_{l, m}(\theta, \varphi)=j^{l} j_{l}\left(k_{0} R\right) Y_{l, m}(\theta, \varphi),
$$

relying on an expansion of an individual far-field pattern into spherical harmonics

$$
\begin{gathered}
e^{j k_{0} \mathbf{r} \cdot \mathbf{p}^{\prime}}=4 \pi \sum_{l^{\prime}, m^{\prime}} j^{l^{\prime}} j_{l^{\prime}}\left(k_{0} R\right) Y_{l^{\prime}, m^{\prime}}(\theta, \varphi) Y_{l^{\prime}, m^{\prime}}^{*}\left(\theta^{\prime}, \varphi^{\prime}\right) \\
\text { with } \quad l^{\prime}=0,1,2, \ldots,+\infty \quad \text { and } \quad\left|m^{\prime}\right| \leq l^{\prime},
\end{gathered}
$$

where $j_{l}\left(k_{0} R\right)$ is the spherical Bessel function of the first kind of order $l$. Note that the far-field pattern has the same angular variation as the excitation and is proportional to the spherical Bessel function. Let $L$ be the highest order mode that can be excited by the array at a reasonable strength. A rule of thumb for determining $L$ is $L=\left\lceil k_{0} R\right\rceil$, since $j_{l}\left(k_{0} R\right)$ decays exponentially when $l$ exceeds its argument $k_{0} R$. The spherical harmonics $Y_{l, m}(\theta, \varphi)$ do not exhibit an exponential increase as a function of $l$, thus for spherical phase modes of order $l>L$ and $|m| \leq l, f_{l, m}(\theta, \varphi)$ is small. The number of spherical phase modes that can be excited at a reasonable strength is thus $(L+1)^{2}$.

\section{SPHERICAL PHASE MODE EXCITATION WITH A SAMPLED APERTURE}

For an $N$ element spherical array, the normalized beamforming weight vector to excite the array with phase mode $(l, m)$, $l \leq L$ and $|m| \leq l$, is a sampled version of $w_{l, m}\left(\theta^{\prime}, \varphi^{\prime}\right)=$ $Y_{l, m}\left(\theta^{\prime}, \varphi^{\prime}\right)$

$$
\begin{array}{r}
\mathbf{w}_{l, m}^{H}=\frac{4 \pi}{N}\left[w_{l, m}^{1} Y_{l, m}\left(\theta_{1}, \varphi_{1}\right), w_{l, m}^{2} Y_{l, m}\left(\theta_{2}, \varphi_{2}\right),\right. \\
\left.\ldots, w_{l, m}^{N} Y_{l, m}\left(\theta_{N}, \varphi_{N}\right)\right] .
\end{array}
$$

For mode orders $l \leq L$, the resulting array pattern can be expressed as

$$
\begin{aligned}
f_{l, m}^{s}(\theta, \varphi)= & \frac{4 \pi}{N} \sum_{n=1}^{N} w_{l, m}^{n} Y_{l, m}\left(\theta_{n}, \varphi_{n}\right) e^{j k_{0} \mathbf{r} \cdot \mathbf{p}_{n}} \\
= & 4 \pi \sum_{l^{\prime}, m^{\prime}} j^{l^{\prime}} j_{l^{\prime}}\left(k_{0} R\right) Y_{l^{\prime}, m^{\prime}}(\theta, \varphi) \\
& \cdot \sum_{n=1}^{N} \frac{4 \pi}{N} w_{l, m}^{n} Y_{l, m}\left(\theta_{n}, \varphi_{n}\right) Y_{l^{\prime}, m^{\prime}}^{*}\left(\theta_{n}, \varphi_{n}\right)
\end{aligned}
$$

The superscript $s$ denotes sampled aperture. We desire that the spherical array with a discrete aperture behaves in a similar fashion as a spherical array with a continuous aperture. In order that a spherical array with a discrete aperture behaves like a spherical array with continuous aperture, it is necessary that

$$
\begin{array}{r}
\frac{4 \pi}{N} \sum_{n=1}^{N} w_{l, m}^{n} Y_{l, m}\left(\theta_{n}, \varphi_{n}\right) Y_{l^{\prime}, m^{\prime}}^{*}\left(\theta_{n}, \varphi_{n}\right)=\delta_{l-l^{\prime}} \delta_{m-m^{\prime}} \\
\forall l, l^{\prime} \leq L \quad \text { and } \quad|m|,\left|m^{\prime}\right| \leq l,
\end{array}
$$

where $\delta_{n}$ denotes a Kronecker delta function at $n=0$. To obtain this goal, we still have the positions of the antenna elements $\left(\theta_{n}, \varphi_{n}\right)$ and the weights $w_{l, m}^{n}$ at our disposal. In the particular case of a circular array, the antenna elements are placed uniformly over the aperture. There exists a minimal number of antenna elements which are required such that the array behaves like a circular array with a continuous aperture. This minimal number of antennas only depends on the dimensions of the array [3]. For a spherical array, similar conditions are valid. However, there only exists a limited number of configurations that sample a sphere uniformly. These configurations correspond to the vertices of the platonic solids. Hardin and Sloane [5] proposed the nearly uniform sampling of a sphere, which offers a much wider range of array configurations. In combination with equal sampling weights, it is equired that for nearly uniform sampling

$$
\frac{4 \pi}{N} \sum_{n=1}^{N} Y_{l^{\prime}, m^{\prime}}^{*}\left(\theta_{n}, \varphi_{n}\right) Y_{l, m}\left(\theta_{n}, \varphi_{n}\right)=\delta_{l-l^{\prime}} \delta_{m-m^{\prime}}
$$

is valid for $l+l^{\prime}<L_{\max }$, where $L_{\max }$ depends on the number of sample points $N$. In order that all significant spherical phase modes $(l \leq L)$ can be excited correctly, it is necessary that $2 L \leq L_{\max }$. There exists a minimal number of antenna elements, wherefore $L_{\max }=2 L$.

\section{SPHERICAL ESPRIT ALGORITHM}

We consider a spherical array with $N$ antenna elements. Only spherical phase modes of order $l \leq L$ can be excited at reasonable strength. The number of antenna elements $N$ is chosen such that the corresponding $L_{\max } \geq 2 L$. The discussion of Section 4 dictates that the beamforming matrix $\mathbf{V}^{H}$, where

$$
\begin{aligned}
\mathbf{V}= & {\left[\mathbf{V}_{0} \vdots \mathbf{v}_{1} \vdots \ldots \vdots \mathbf{V}_{L}\right], } \\
& \mathbf{V}_{l}=\sqrt{\frac{N}{4 \pi}}\left[\mathbf{w}_{l,-l} \vdots \ldots \vdots \mathbf{w}_{l,-1} \vdots \mathbf{w}_{l, 0} \vdots \mathbf{w}_{l, 1} \vdots \ldots \vdots \mathbf{w}_{l, l}\right],
\end{aligned}
$$

and $\mathbf{w}_{l, m}$ is defined in (6), performs the following mapping

$$
\mathbf{a}_{\mathbf{o}}(\theta, \varphi)=\mathbf{V}^{H} \mathbf{a}(\theta, \varphi)=\left[\mathbf{a}_{\mathbf{o}, 0}(\theta, \varphi)^{T} \vdots \ldots \vdots \mathbf{a}_{\mathbf{o}, L}(\theta, \varphi)^{T}\right]^{T}
$$


where

$$
\begin{aligned}
& \mathbf{a}_{\mathbf{o}, l}(\theta, \varphi)=\sqrt{4 \pi N} j^{l} j_{l}\left(k_{0} R\right) \mathbf{Y}_{l}(\theta, \varphi) \\
& \quad=\sqrt{4 \pi N} j^{l} j_{l}\left(k_{0} R\right)\left[Y_{l,-l}(\theta, \varphi), \ldots, Y_{l, l}(\theta, \varphi)\right]^{T} .
\end{aligned}
$$

Consider extracting three subvectors of size $L^{2}$ from the spherical phase mode manifold $\mathbf{a}_{\mathbf{0}}(\theta, \varphi)$, as $\mathbf{a}^{(i)}(\theta, \varphi)=$ $\Delta^{(i)} \mathbf{a}_{\mathbf{0}}(\theta, \varphi), i=-1,0,1$, where the selection matrix $\Delta^{(i)}$ acts on each subvector $\mathbf{a}_{\mathbf{0}, l}(\theta, \varphi)$ (defined in (12)) of $\mathbf{a}_{\mathbf{0}}(\theta, \varphi)$. The selection matrices $\Delta^{(-1)}, \Delta^{(0)}$ and $\Delta^{(1)}$ select the first, middle, and last $2 l-1$ elements from $\mathbf{a}_{\mathbf{o}, l}(\theta, \varphi)$. It is clear that $\Delta^{(i)} \mathbf{a}_{\mathbf{0}, 0}(\theta, \varphi)=0$. The three subvectors $\mathbf{a}^{(i)}(\theta, \varphi)$ can be related to each other by a recursive relationship between spherical harmonics.

$$
\begin{aligned}
2 m Y_{l, m}(\theta, \varphi)+ & \lambda_{l m}^{+} Y_{l, m+1}(\theta, \varphi) \tan \theta e^{-j \varphi} \\
& +\lambda_{l m}^{-} Y_{l, m-1}(\theta, \varphi) \tan \theta e^{j \varphi}=0,
\end{aligned}
$$

where $\lambda_{l m}^{ \pm}=\sqrt{(l \mp m)(l \pm m+1)}$. This leads to the relationship:

$$
2 \boldsymbol{\Gamma} \mathbf{a}^{(0)}+\boldsymbol{\Lambda}^{+} \mathbf{a}^{(1)} \mu^{*}+\boldsymbol{\Lambda}^{-} \mathbf{a}^{(-1)} \mu=0,
$$

where $\mu=\tan \theta e^{j \varphi}$. The $L^{2} \times L^{2}$ diagonal matrices $\boldsymbol{\Gamma}$ and $\Lambda^{ \pm}$are defined by

$$
\boldsymbol{\Gamma}=\operatorname{diag}\{0,-1,0,1, \ldots,-L+1, \ldots, L-1\}
$$

and

$$
\begin{aligned}
\mathbf{\Lambda}^{ \pm}=\operatorname{diag}\left\{\lambda_{1,0}^{ \pm}, \lambda_{2,-1}^{ \pm},\right. & \lambda_{2,0}^{ \pm}, \lambda_{2,1}^{ \pm}, \ldots \\
& \left., \lambda_{L,-L+1}^{ \pm}, \ldots, \lambda_{L, L-1}^{ \pm}\right\} .
\end{aligned}
$$

Let $\mathbf{A}=\left[\mathbf{a}_{\mathbf{o}}\left(\theta_{1}, \varphi_{1}\right), \mathbf{a}_{\mathbf{o}}\left(\theta_{1}, \varphi_{1}\right), \ldots, \mathbf{a}_{\mathbf{0}}\left(\theta_{P}, \varphi_{P}\right)\right]$ be the $(L+$ $1)^{2} \times P$ DOA matrix in the $(L+1)^{2}$-dimensional spherical phase mode space, where $P$ is the number of sources incident on the spherical array. Furthermore, let $\mathbf{A}^{(i)}=\Delta^{(i)} \mathbf{A}$, $i=-1,0,1$, where the selection matrices $\Delta^{(i)}$ were defined previously. Relying on (14), $\mathbf{A}^{(-1)}, \mathbf{A}^{(0)}$ and $\mathbf{A}^{(1)}$ are related by

$$
2 \boldsymbol{\Gamma} \mathbf{A}^{(0)}+\boldsymbol{\Lambda}^{+} \mathbf{A}^{(1)} \boldsymbol{\Phi}^{*}+\boldsymbol{\Lambda}^{-} \mathbf{A}^{(-1)} \mathbf{\Phi}=0,
$$

where

$$
\boldsymbol{\Phi}=\operatorname{diag}\left\{\mu_{1}, \ldots, \mu_{P}\right\} .
$$

Let $\mathbf{S}$ be an $(L+1)^{2} \times P$ matrix whose columns span $\mathcal{R}\{\mathbf{A}\}$ such that $\mathbf{S}=\mathbf{A T}$, where $\mathbf{T}$ is an unknown $P \times P$ matrix. $\mathbf{S}$ can be obtained from an EVD of the covariance matrix formed in phase mode space. Let $\mathbf{S}^{(i)}=\Delta^{(i)} \mathbf{S}, i=-1,0,1$. It follows from (17) that $2 \boldsymbol{\Gamma} \mathbf{S}^{(0)}+\boldsymbol{\Lambda}^{+} \mathbf{S}^{(1)}\left(\mathbf{T}^{-1} \boldsymbol{\Phi}^{*} \mathbf{T}\right)+$ $\boldsymbol{\Lambda}^{-} \mathbf{S}^{(-1)}\left(\mathbf{T}^{-1} \mathbf{\Phi} \mathbf{T}\right)=0$. Rewriting this equation in block matrix form, we have

$$
2 \Gamma \mathbf{S}^{(0)}+\mathbf{E} \underline{\Psi}=0
$$

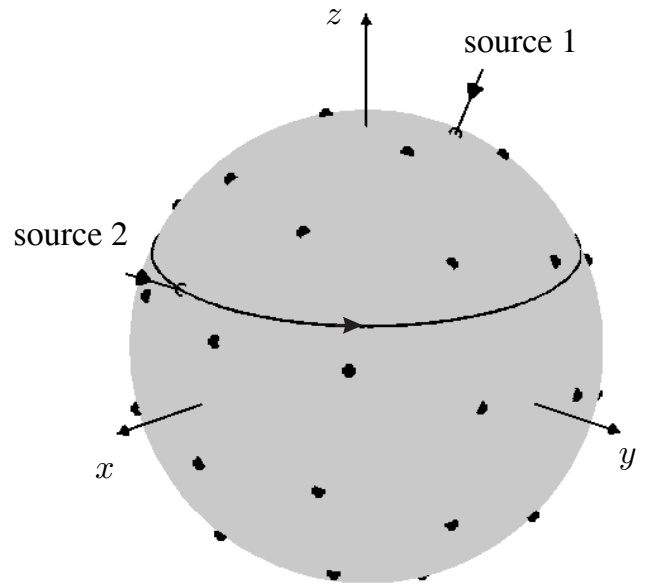

Fig. 1. Spherical array configuration of 36 antennas with two sources arriving at the sphere.

where $\quad \mathbf{E}=\left[\boldsymbol{\Lambda}^{-} \mathbf{S}^{(-1)}: \boldsymbol{\Lambda}^{+} \mathbf{S}^{(1)}\right] \quad$ and $\quad \underline{\boldsymbol{\Psi}}=\left[\begin{array}{c}\mathbf{T}^{-1} \mathbf{\Phi} \mathbf{T} \\ \mathbf{T}^{-1} \mathbf{\Phi}^{*} \mathbf{T}\end{array}\right]$.

Note, $\mathbf{E}$ is a $L^{2} \times 2 P$-matrix and $\boldsymbol{\Psi}$ is a $2 P \times P$-matrix. When $L^{2}>2 P,(19)$ is overdetermined and can be shown to have a unique solution $\underline{\Psi}$. One can thus solve (19) for $\underline{\boldsymbol{\Psi}}$ and compute the eigenvalues of either the top or bottom $P \times P$ subblock to determine $\mu_{i}=\tan \theta_{i} e^{j \varphi_{i}}, i=1, \ldots, P$. The estimates of the elevation and azimuth angles of the $i$ th source follow from $\widehat{\theta}_{i}=\tan ^{-1}\left|\widehat{\mu}_{i}\right|$ and $\widehat{\varphi}_{i}=\arg \left(\widehat{\mu}_{i}\right)$, respectively. The algorithm based on this development, spherical ESPRIT, is closed form and provides automatically paired azimuth and elevation estimates.

\section{SIMULATIONS}

We demonstrate the efficacy of spherical ESPRIT by some simulations. A spherical array of radius $R=\lambda / 3$ was employed. This means that spherical phase modes up to $L=$ $\left\lceil k_{0} R\right\rceil=3$ can be excited at a reasonable strength. Consider $N=36$ antenna elements in the spherical array. For this configuration $L_{\max }=8>2 L$, such that this array behaves like a continuous spherical aperture. Two equipowered sources in the far field of the array with arrival angles $\left(\theta_{1}, \varphi_{1}\right)=\left(23^{\circ}, 150^{\circ}\right)$ and $\left(\theta_{2}, \varphi_{2}\right)=\left(65^{\circ}, \alpha\right)$ were considered. The azimuth angle of the second source is varied from $0^{\circ}$ to $360^{\circ}$ in steps of $20^{\circ}$. The sources were highly correlated with a correlation coefficient of $\rho=0.9 e^{j \pi / 4}$ at the array center. The number of snapshots was $K=64$ and sample statistics were computed from 200 independent trials. The SNR level was $20 \mathrm{~dB}$, where the SNR quoted is per source per array element. In Fig. 1 the antenna array configuration is shown, where the 36 antennas are distributed nearly 

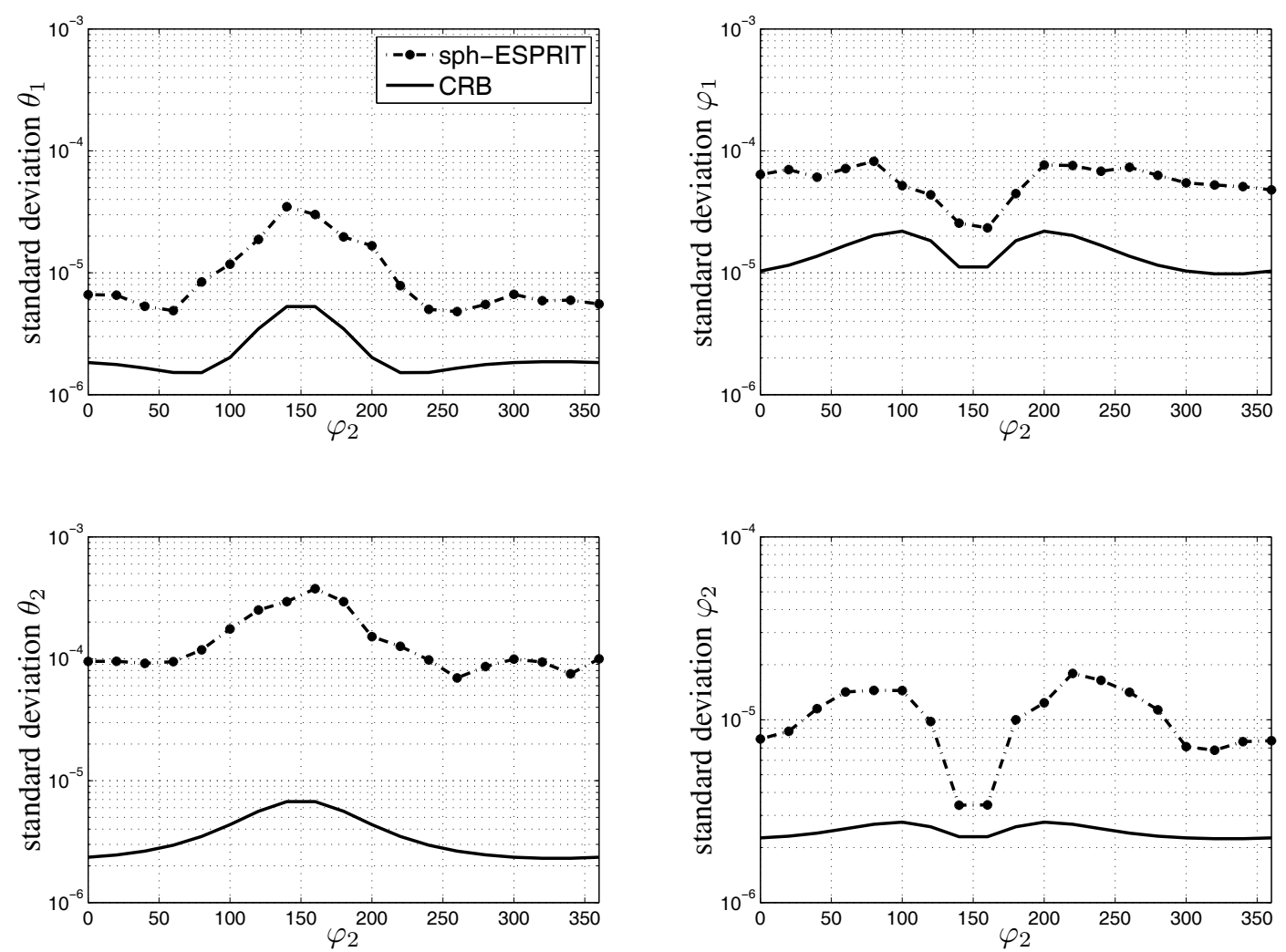

Fig. 2. Performance of the spherical ESPRIT estimators.

uniformly over the sphere. The two directions-of-arrival of the impinging sources are also indicated. Notice that, since the second source has a varying azimuth angle, the distance between the DOAs of the two sources varies. In Fig. 2 we observe the standard deviations (in radians) of the estimated angles-of-arrival. The azimuth angles of the sources are estimated with the highest accuracy when the sources are close to each other. For the elevation estimates it is just the opposite. In Fig. 2 the corresponding Cramer Rao bounds for the estimated parameters are also plotted. It is clear that the spherical ESPRIT algorithm is not capable to deliver DOA estimates with an accuracy that is very close to the corresponding CRBs. For ESPRIT-based algorithms, such as ESPRIT and UCA-ESPRIT, this conclusion is generally valid. It is clear that the proposed algorithm is remarkable for its computational simplicity, but this affects the ultimate accuracy of the estimates. This has to be put into perspective, because in the considered simulated example the most inaccurate estimate (estimate of $\theta_{2}$ when $\varphi_{2}=150^{\circ}$ ) has a mean error of $3.4^{\circ}$, which is only a fraction of the $3 \mathrm{~dB}$ beamwidth of the cophasal beam steered spherical array.

\section{REFERENCES}

[1] R.O. Schmidt, "Multiple emitter location and signal parameter estimation," IEEE Trans. Antenn. Propagat., vol. 34, pp. 276-280, March 1986.

[2] R. Roy, A. Paulraj, and T. Kailath, "ESPRIT- a subspace rotation approach to estimation of parameters of cisoids in noise," IEEE Trans. Acoust., Speech, Signal Processing, vol. 34, no. 5, pp. 1340 - 1342, Oct. 1986.

[3] C.P. Mathews and M.D. Zoltowski, "Eigenstructure techniques for 2-d angle estimation with uniform circular arrays," IEEE Trans. on signal processing, vol. 42, no. 9, pp. 2395 - 2407, Sept. 1994.

[4] E. De Witte, H.D. Griffiths, and P.V. Brennan, "Phase mode processing for spherical arrays," Electronics Letters, vol. 39, no. 20, pp. 1430-1431, Oct. 2003.

[5] R.H. Hardin and N.J.A Sloane, “Mclaren's improved snub cube and other new spherical designs in three dimensions," Discrete computational geometry, vol. 15, pp. 429 - 441, 1995. 


\title{
2008 IEEE International \\ Conference on Acoustics, Speech, and Signal Processing
}

\author{
Proceedings
}

March 30-April 4, 2008

Caesars Palace

Las Vegas, Nevada, U.S.A.

Sponsored by

The Institute of Electrical and Electronics Engineers

Signal Processing Society

IEEE Catalog Number: 08CH37930

ISBN: 1-4244-1484-9
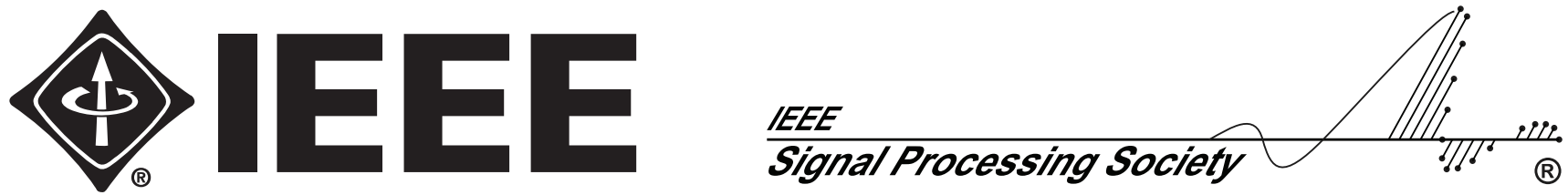